\title{
PROCESADO TÉRMICO DE MOLDES CONFECCIONADOS CON REVESTIMIENTO DE JOYERÍA MEDIANTE MICROONDAS POR CALENTAMIENTO HÍBRIDO
}

\author{
Francisco Moreno Candel*, Fátima Felisa Acosta Hernández**, \\ Itahisa Pérez-Conesa*** y José Antonio Aguilar Galea
}

\section{RESUMEN}

Esta investigación pretende contribuir a la comprensión y control del procesamiento de materiales implicados en la fundición artística mediante microondas, con el fin de facilitar este tipo de fuentes de energía en los procesos térmicos de descere. Concretamente se estudió el comportamiento de pequeñas muestras confeccionadas con revestimientos refractarios comerciales sobre modelos sencillos de cera. Se diseñó una adaptación y compatibilización de la técnica convencional de joyería fundida para validar un tratamiento integral con esta alternativa de energía. De un lado, se diseñó una curva de tiempo/potencia adecuada para el descere y secado inicial de estas pastas, poniendo en valor las propiedades dieléctricas del agua presente su confección. De otro, se estudió un procedimiento factible para el desarrollo de su quemado, a través del empleo de calentamiento híbrido utilizando el carburo de silicio $(\mathrm{SiC}-\beta)$ como susceptor. Para aplicar una temperatura escalonada, se ha fabricó una doble mufla que combina materiales transparentes y absorbentes con la capacidad de alcanzar $750^{\circ} \mathrm{C}$ con potencia MW de 700w. Con los resultados obtenidos se intensificó el interés por estudiar un procesamiento térmico para el descere y cocción de los revestimientos refractarios con modelos más grandes y formas más complejas orientadas a la joyería y escultura de pequeño formato. Palabras clave: fundición artística, joyería, tecnología microondas, procedimientos escultóricos.

\section{THERMAL PROCESSING OF MOLDS MADE WITH JEWELLERY COATING BY MICROWAVE THROUGH HYBRID HEATING}

\section{Abstract}

This research aims to contribute to the understanding and control of the processing of materials involved in artistic casting by microwaves, in order to facilitate this type of energy sources in the thermal processes of dewaxing. Specifically, the behavior of small samples made with commercial refractory coatings on simple wax models was studied. An adaptation and compatibilization of the conventional technique of fused jewelry was designed to validate an integral treatment with this energy alternative. On the one hand, a suitable time/power curve was designed for the initial dewaxing and drying of these pastes, taking into account the dielectric properties of the water present in their confection. On the other hand, a feasible procedure was studied for the development of their burning, through the use of hybrid heating using silicon carbide $(\mathrm{SiC}-\beta)$ as a susceptor. In order to apply a step temperature, a double muffle combining transparent and absorbing materials with the capacity to reach $750^{\circ} \mathrm{C}$ with MW power of $700 \mathrm{w}$ was fabricated. The results obtained intensified the interest in studying a thermal processing for the dewaxing and firing of refractory linings with larger models and more complex shapes oriented to jewelry and small format sculpture.

KEYwORDs: melting casting, jewellery, microwave technology, sculptural procedures.

DOI: https://doi.org/10.25145/j.bbaa.2021.15.04

Revista Bellas Artes, 15; diciembre 2021, pp. 89-107; ISSN: e-2530-8432 


\section{INTRODUCCIÓN}

Desde los inicios de la fundición de metales se ha investigado en torno a facilitar, economizar y mejorar la calidad de sus procesos valiéndose de la tecnología y los materiales ${ }^{1}$. Las dificultades de producción que encuentran los métodos artesanales en el mercado en confrontación con cadenas de producción repetitivas y mecanizadas son numerosas, volviéndose estas últimas muy competitivas. De tal competencia se justifica que, concretamente, la joyería y la escultura en metal fundido deba reinventarse de manera continuada hacia la simplificación de sus metodologías, permitiendo así que tanto artistas como profesionales encuentren facilidades para generar obra en metal.

La técnica más generalizada en el sector de la joyería fundida es la «cera perdida». De tradición milenaria, se basa en el empleo de la cera para crear un modelo, que es recubierto de material refractario, que en el ámbito de la joyería se vierte sobre un encofrado de forma cilíndrica, que contiene el modelo en cera que se pretende reproducir en metal. Una vez que ha fraguado este molde, se debe hornear para evacuar la cera que contiene, de ahí la denominación de "cera perdida», y conseguir el hueco necesario para verter el metal fundido. En esta misma secuencia los moldes son deshidratados para evitar las reacciones que se producirían al contacto del metal fundido con un material con humedad. Por estas razones los cilindros deben ser termorresistentes, ya que serán horneados durante el tratamiento ${ }^{2}$.

Estos compuestos son preparados en estado líquido, de manera que se adhieren a los modelos de cera registrando a la perfección los detalles de cualquier forma. Tras su fraguado, se realiza el tratamiento térmico, donde se produce primero la evacuación de la mayor parte de la cera por derretimiento y, posteriormente, la cocción del molde entre $750^{\circ} \mathrm{C}$ y $850^{\circ} \mathrm{C}$, quedando apto para la recepción del metal fundido ${ }^{3}$. Los revestimientos refractarios requieren en su elaboración de la incorporación de un porcentaje importante de agua que es esencial para hidratar el yeso, su principal componente, resultando, una vez fraguado, un molde muy húmedo, con un $40 \%$ de parte acuosa. Razón por la cual precisan perder esta humedad de manera lenta y progresiva para evitar la aparición de grietas debido a las tensiones del secado. Pues esto supondría una inestabilidad que le haría descomponerse, producir fugas o errores tras la fundición. En el procedimiento tradicional, se aplican rampas de potencia de $0^{\circ} \mathrm{C}$ hasta $800^{\circ} \mathrm{C}$ durante 8 horas. Realizándose mesetas

* Universidad de La Laguna. E-mail: pacomorenocandel@gmail.com.

** Universidad de La Laguna.E-mail: ffacosta@ull.edu.es.

*** Universidad de La Laguna. E-mail: iperecon@ull.edu.es.

$\diamond$ Universidad de Sevilla.E-mail: jantonio@us.es.

1 A. Sorroche Cruz, I. Lozano Rodríguez, J. Durán Suárez, J. Peralbo Cano y C. Bellido Márquez. «Mejora de los métodos de trabajo en la fundición escultórica con modelos de poliestireno expandido». Revista Técnica Industrial, 1-2 (2009).

2 M. Groover. «Fundamentos de manufactura moderna». México, 243 (1997).

3 J. Corredor. Técnicas de fundición artística. Universidad de Granada, 1997. 
graduales de $100 \%$, lo cual lo convierte en un sistema largo y costoso ${ }^{4}$. El objetivo es aumentar la temperatura paulatinamente para que, a la misma vez que se va eliminando cualquier residuo de cera, se proceda al secado sin que se vea perjudicada la integridad de los moldes. De este modo, el material se compacta cohesionando su composición molecularmente para generar un molde resistente, que asegure una colada del metal eficiente y sin peligrosidad de reacciones 5 .

Los dispositivos utilizados habitualmente en los talleres actuales son hornos eléctricos diseñados para cocer cerámicas. Generalmente se trata de hornos aparatosos y que generan un alto consumo energético para alcanzar ese intervalo de temperatura en unas horas. Además, normalmente la infraestructura específica para la producción de piezas tan precisas y delicadas como las que se conciben en joyería es cara y sofisticada, aunque resulta duradera y efectiva. Por tanto, la necesidad de simplificar las instalaciones y los procesos de producción en este ámbito resulta ineludible, tanto para talleres profesionales como para pequeńos productores o artesanos.

Concretamente, en esta investigación se plantea un estudio de viabilidad, que tiene como objetivo sustituir los hornos convencionales eléctricos por otros basados en la energía MW para la aplicación de los tratamientos térmicos de los moldes de revestimiento de base de yeso en la joyería. Para ello, es necesario estudiar su comportamiento y adaptar el procedimiento al calentamiento por $\mathrm{MW}$, con el fin de posibilitar esta tecnología y así formular ventajas al sector.

El alto contenido en agua de las pastas refractarias funciona como vehículo termoconductor para llevar a cabo la primera parte de la investigación. El calentamiento dieléctrico favorece la eliminación de la humedad a través de la irradiación de las microondas. El agua, al estar compuesta por moléculas polares, actúa como un material absorbente de microondas. Cuando interactúa con esta fuente de energía, los dipolos chocan entre sí provocando calor transferido por conducción a través de la red cristalina. De este modo, aumenta la temperatura del conjunto del material expuesto. Partiendo de esta premisa, se puede llevar a cabo un secado inmediato, rápido y uniforme. Se consiguen así perfiles de humedad uniformes a lo largo del material, evitando que haya gradientes de temperatura en la pieza que pudieran generar fallas por contracción diferencial ${ }^{6}$. El aumento de temperatura gradual para un descerado y secado sin inconvenientes depende del tiempo de exposición a la radiación y potencia de las MW emitidas. Por otro lado, se palían los problemas producidos por el cambio que presentan los materiales en sus propiedades dieléctricas al aumentar su temperatura, ya que esto supone un inconveniente en el proceso de secado de los moldes con esta tecnología. A medida que los negativos aumentan su temperatura pierden la humedad y, por lo tanto, el calenta-

${ }^{4}$ J. Benavente. La fundición a la cera perdida: Microfusión. Barcelona: Alsina, 1992.

5 M. Groover. Fundamentos...

${ }^{6}$ F.J. Ramírez Gil. «Comparación entre las técnicas convencionales y las microondas para la conformación y secado de materiales cerámicos fabricados por colado». Colombia 16 (2010).

7 J. Menéndez y A.H. Moreno. «Aplicaciones del calentamiento con energía microondas». En Secado industrial con microondas (85). Cotopaxi: Ed. Universidad Técnica de Cotopaxi, 2017. 
miento dieléctrico pierde eficacia, imposibilitando que el cuerpo alcance la temperatura necesaria. Para ello, se propone hacer uso del calentamiento híbrido ${ }^{8}$, para el que se hacen necesarios los susceptores, es decir, materiales absorbentes por MW. David Reid ${ }^{9}$ trabajó en base a un sistema experimental para fundir piezas de plata en un microondas doméstico. Añadía magnetita como material susceptor a la composición de una mufla con la que conseguía aumentar la temperatura para fundir pequeñas piezas de metal ${ }^{10}$. Partiendo de su propuesta, en este estudio, se incluye el uso del carburo de silicio $(\mathrm{SiC}-\beta)^{11}$ como catalizador aplicado a una mufla propiamente diseñada para introducir los moldes cilíndricos y conseguir el aumento de temperatura hasta $750^{\circ} \mathrm{C}$, aproximadamente. De este modo, se adapta el proceso de descere, secado y deshidratación de moldes para la fundición de joyería a esta tecnología como fuente de energía.

Los resultados de esta investigación pretenden contribuir a la mejora de los procedimientos, simplificando los procesos característicos de fundición artística con el fin de lograr mejoras sustanciales en tiempo, rentabilidad y sostenibilidad. Concretamente, se pretende facilitar el acceso a esta tecnología, es decir, el procesamiento térmico de los moldes, a talleres profesionales, docentes y artistas plásticos que pretendan abordar la producción de obra artística en metal fundido.

\section{ANTECEDENTES}

En la actualidad, la tecnología MW resulta una potente herramienta en multitud de aplicaciones industriales. Su empleo en procesos productivos ha cobrado mucho interés gracias a la posibilidad de reducción de los costes y tiempos. Inicialmente, eran tan sólo utilizadas en los productos alimenticios, pero se han ido extendiendo a la ciencia y la tecnología de materiales, como se puede comprobar consultando la literatura científica y el incremento de entradas bibliográficas sobre las microondas ${ }^{12}$.

Los sistemas de calentamiento convencionales transmiten el calor a través de los mecanismos de conducción, radiación y convección, desde la superficie de la pieza hacia el interior, mientras que el calentamiento con microondas se produce por una absorción selectiva de energía de las ondas electromagnéticas desde el interior hacia

${ }^{8}$ Y. Segura de Jesús, \& G. Carbaja. «El uso de radiación de microondas para la síntesis de Nanopartículas». Revista de Innovación Sistemática, 1(2), (2017): 46-56.

9 D. Reid. «El primer vaciado fundido (en plata) por microondas se realizó en 1993. Se demostró la técnica en una conferencia sobre microondas en el St. John's College de Cambridge en 1995». (2018). https://hackaday.com/2005/01/24/microwave-oven-foundry/n. Artes, 4, (2006):13-28.

${ }_{10}$ J.C. Albaladejo González. «Fundición a la cera perdida: Cellini y la magnetita». Bellas

${ }_{11}$ J.A. Aguilar Galea. «La enseñanza de la fundición artística en las facultades de Bellas Artes Españolas». Tesis doctoral. Universidad de Sevilla, 2000.

12 R. Buenavente Martínez. Desarrollo de materiales cerámicos avanzados con altas prestaciones mediante técnicas no convencionales. Valencia: Universidad Politécnica de Valencia, 2015. 
la superficie de la pieza. Este hecho permite un calentamiento más uniforme, eficaz y rápido, aprovechando toda la energía en el cuerpo a calentar ${ }^{13}$. Sin embargo, pese a ser muy efectiva, tiene ciertas particularidades que se deben conocer para explotar sus capacidades. Las razones son varias: por un lado, el diseño de estos sistemas resulta algo complejo, requiriendo en muchas ocasiones el conocimiento de varios campos. Y por otro, el funcionamiento del calentamiento dieléctrico está en constante investigación, lo que puede fomentar un sentimiento de rechazo por falta de comprensión ${ }^{14}$. Este calentamiento se basa en la diferencia de materiales, agrupándose en tres tipos: transparentes, opacos y absorbentes. Los primeros no son alterados, dejando pasar las microondas libremente a través de su estructura. Los opacos no permiten el paso de las microondas reflejándolas. Y los absorbentes captan la energía de las microondas y la convierten en calor ${ }^{15}$. Los materiales absorbentes MW aceleran los procesos térmicos gracias a una alta conductividad, dependiendo fundamentalmente de las pérdidas por conducción. Un material es un buen susceptor de energía dependiendo de cuánta y a qué velocidad puede absorber dicha energía para transformarla en calor ${ }^{16}$.

Los sistemas de procesado térmico a través de MW están en continua exploración para aplicarlos a diferentes ámbitos. Según McCabe (1998) «El secado es, posiblemente, la más antigua, común y diversa de las operaciones unitarias de la ingeniería química... Consiste en la operación de separación, total o parcial, del líquido que lo acompaña, con el objetivo de reducir el contenido del mismo hasta un valor aceptable» ${ }^{17}$.

El secado con microondas se basa en la energía adicional absorbida por los líquidos presentes en el proceso. Debido a las propiedades que posee el agua y a medida que se va produciendo el calentamiento dieléctrico, se origina la evaporización y, en consecuencia, el secado de los moldes cerámicos al ser expuestos a la energía electromagnética $(300 \mathrm{Mhz}-300 \mathrm{GHz})^{18}$. Por ello, en las aplicaciones de secado se produce un efecto de calentamiento selectivo. Pues la absorción de microondas es mayor y más eficaz en zonas donde hay más humedad, resultando un secado intensivo pero uniforme, obteniendo mayor calidad en los resultados ${ }^{19}$. Los beneficios que más interesan del uso de las microondas frente a las técnicas de secado convencionales son la reducción en el tiempo de secado y el descenso de consumo

13 A.C. Álvarez. Sinterización en aplicaciones industriales del calentamiento con energía microondas. Ecuador: Universidad Técnica de Cotopaxi, 2017.

14 P.J. Plaza González. Control de la temperatura en sistemas de calentamiento por microondas. Valencia: UPV, 2015.

${ }^{15}$ F.J. Ramírez Gil. «Comparación entre las técnicas convencionales y las microondas para la conformación y secado de materiales cerámicos fabricados por colado». Colombia 16 (2010). (2011): $129-136$.

${ }^{16} \mathrm{~J}$. Prado Gonjal y E. Morán. Sintesis asistida por microondas de sólidos inorgánicos, 2

${ }_{17}$ Citado en J. Menéndez y A.H. Moreno. Aplicaciones...

${ }^{18}$ C. Cárdenas, Restrepo et al. Las microondas como una alternativa para el secado de materiales cerámicos tradicionales. Universidad Simón Bolívar, 2009.

${ }_{19}$ J. Menéndez y A.H. Moreno. Aplicaciones... 
energético. Algunos estudios muestran una reducción del 33\% en tiempos de secado con respecto al calentamiento convencional, manteniéndose, sin embargo, la resistencia mecánica ${ }^{20}$.

Como dijimos, el antecedente principal del uso del microondas en fundición artística lo personaliza David Reid ${ }^{21}$, que inicia una línea de investigación que conocemos gracias a Juan Carlos Albaladejo González, catedrático de la Facultad de Bellas Artes de la Universidad de La Laguna. Albaladejo parte de la experimentación sobre la energía microondas del neozelandés, aunque la focaliza en su aplicación en el proceso de descere de la cáscara cerámica ${ }^{22}$. Una investigación que formaliza en proyecto ${ }^{23}$ siendo aprobada en el Plan nacional de I+D+I en 2011, bajo el título «Alternativas al descere en la fundición de cascarilla cerámica (Ceramic Shell Casting): Técnica por microondas». Este trabajo abarcó tres años que se prorrogaron con otros dos en otro proyecto bajo la misma denominación que se desarrolló del 2017 al 2019.

Aunque realmente la investigación sobre las posibilidades de la energía MW aplicada al proceso de descere en fundición artística las inicia este investigador mucho antes, dado que ya en 2006 sale a la luz un artículo que publica en la revista Bellas Artes, titulado: «Fundición a la Cera Perdida: Cellini y la magnetita». En ésta, referencia a su vez a David Reid como precursor del uso del microondas en fundición: «David Reid tiene un sistema experimental de microfusión con microondas. Se basa en la capacidad de absorción de la magnetita a las microondas. Incorporando magnetita a la composición del molde consigue calentarlo lo suficiente para fundir pequeñas piezas de plata en un horno microondas doméstico $»^{24}$.

Reid de esta forma planteó el fundir metales en un microondas corriente, aportando la clave, la incorporación del susceptor en la composición de la cascarilla, pero deja fuera el aspecto que Albaladejo entiende como trascendental en el tema de la fundición con cascarilla cerámica, el descere. La investigación que inicia el profesor de La Laguna en ese momento tiene como protagonista el binomio cáscara/ cera, dado que el procedimiento de descerado ortodoxo de la cáscara, el choque térmico (Flash Dewaxing), dejaría de ser viable por seguridad en centros educativos.

En este artículo de la revista de Bellas Artes, Juan Carlos plantea los límites técnicos de la misma, es decir, un microondas convencional, significando el punto de partida de los proyectos de $\mathrm{I}+\mathrm{D}+\mathrm{i}$, que condujeron al diseño y construcción de

${ }^{20}$ C. Carolina. «Las microondas como una alternativa para el secado de materiales cerámicos tradicionales». Revista Latinoamericana de Metalurgia y Materiales (2009): 428-431.

${ }^{21}$ Químico metalúrgico neozelandés que gracias a una beca de investigación estudió las técnicas de fundición primitivas por todo el mundo desarrollando la técnica de microfusión con crisol incorporado (conocida como Reid Technique) al aunar estas técnicas con un material nuevo, la cáscara cerámica.

22 J.A. Aguilar Galea. «La enseñanza de la fundición artística en las facultades de Bellas Artes Españolas». Tesis doctoral. Universidad de Sevilla, 2000.

${ }^{23}$ HAR2016-77203-P

${ }^{24}$ J.C. Albaladejo González, «Fundición a la cera perdida: Cellini y la magnetita». Bellas Artes, 4, (2006):13-28 


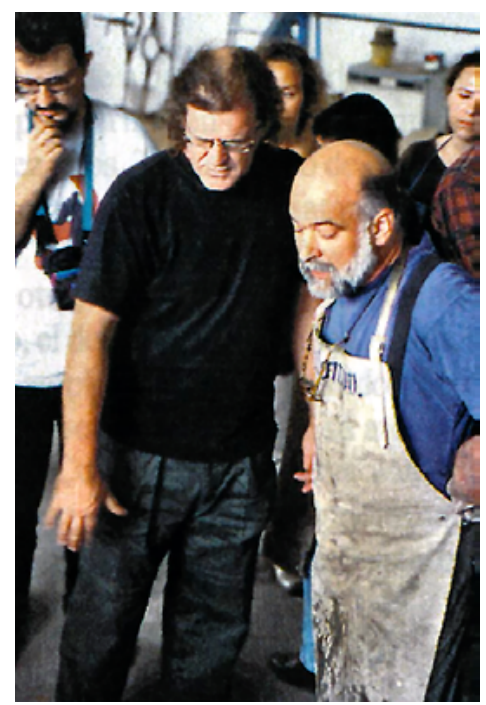

Figura 1. Juan Carlos Albaladejo y David Reid durante la impartición del curso "An investigation into the Properties of Obsidian as a sculptural material», "Una investigación sobre las propiedades escultóricas de la obsidiana», celebrado en la Facultad de Bellas Artes de la Universidad de La Laguna, Tenerife, en 1998. (Aguilar, 2000).

un prototipo de horno microondas para descerar moldes de cascarilla cerámica en el que posteriormente, se estudiaron diferentes tipos de estucos electromagnéticos, siendo sometidos a pruebas de efectividad según rampas de potencia. Estas pruebas protagonizaron la tesis doctoral que, sobre el tema «Técnica del descere por Microondas: Identificación, cuantificación y valoración de los susceptores en el estuco de cascarilla cerámica en la fundición artística», defendiera en 2017 la investigadora Itahisa Pérez Conesa, dirigida por el catedrático, Soledad del Pino ${ }^{25}$ y José Fayos ${ }^{26}$. Estos proyectos condujeron a la patente denominada ES2519990 A1 (07.11.2014), inscrita en colaboración con la Politécnica de Cartagena (30\%) y la ULL (70\%), que «incluye el diseño y fabricación de un horno microondas específico y un procedimiento que perfecciona la fundición de moldes en cascarilla cerámica para obras de arte, joyería, diseño y objetos de pequeño y mediano formato, a la vez que permite ahorrar energía, tiempo y costes $»^{27}$.

${ }_{25}$ Profesora del área de Escultura adscrita al Departamento de Bellas Artes de la Universidad de La Laguna.

${ }^{26}$ Profesor de la Universidad Politécnica de Cartagena. Departamento Tecnologías de la Información y las Comunicaciones. Grupo de Electromagnetismo y Materia.

${ }^{27}$ Pérez Conesa, I. «Técnica del descere por microondas: identificación, cuantificación y valoración de los susceptores». Tesis doctoral, Universidad de La Laguna, 2017. 
Aunque los objetivos específicos de estos trabajos son diferentes a los de esta investigación, el principal objeto de ésta se comparte con sus precedentes, es decir, como se expuso anteriormente, el constituirse como alternativa a los hornos convencionales, basándose en la energía de las microondas para la aplicación de los tratamientos térmicos de los moldes, en este caso de revestimiento cerámico para joyería. Se parte de los resultados y conclusiones de los proyectos, publicaciones y tesis derivados de la línea de investigación que iniciara Juan Carlos Albaladejo para la cáscara cerámica, aunque dirigidos esta vez sobre los moldes de base yeso refractario. Este diferente punto de enfoque distingue los principios de funcionamiento y las referencias de estudio, dado que el sujeto de análisis, la cáscara cerámica, es sustituida por los moldes de revestimiento (yeso refractario), dos materias sustancialmente diferentes con características y metodologías distintas.

\section{MATERIALES Y MÉTODOS}

La metodología puesta en práctica está sujeta a dos objetivos concretos que condicionan el procedimiento de adaptación. De un lado, se pretende descerar el modelo y, del otro, secar el molde, eficazmente, sin que aparezcan signos de deterioro por un calentamiento demasiado agresivo, conservando las características propias del revestimiento en su procesamiento convencional: buena capacidad de registro para reproducir todo tipo de formas y una porosidad adecuada para la extracción de gases. Esto, además de depender de la calidad de la composición de la pasta, que varía según el fabricante, depende irremediablemente de la eficacia de todo el desarrollo preliminar ${ }^{28}$. En consecuencia, se pretende una curva corta y progresiva, controlando la temperatura mediante el escalonamiento del rendimiento del MW, aplicando intervalos variables de tiempo/potencia hasta controlar las altas propiedades dieléctricas del molde por su alto contenido de agua (40\%) o el calentamiento de material susceptor $(\mathrm{SiC})$. Se estudia un procedimiento viable para aumentar la temperatura a $750^{\circ} \mathrm{C}$, una vez perdidas las propiedades dieléctricas por la deshidratación del compuesto, para conseguir el quemado de los restos de cera y evaporación del agua combinada en el molde. Para el cumplimiento de estos objetivos, se diseñan pruebas poniendo en práctica la estrategia ensayo/error y análisis de resultados, que permitan hallar un procedimiento térmico viable.

Para la primera parte del estudio, el descerado, se han elaborado 15 modelos empleando cera roja de modelar de Iberceras (Ref:1950), construidos con una forma simple cilíndrica de $0,50 \mathrm{~mm}$ de diámetro, $50 \mathrm{~mm}$ de longitud y $4 \mathrm{~g}$ de peso, distinguida como Forma A. Estudios precedentes ${ }^{29}$ han determinado que las propieda-

${ }^{28}$ K.A. Krekeler. Microfusión. Fundición con modelo perdido. Gustavo Gili, 1971.

${ }^{29}$ F. Moreno Candel, F.F. Hernández y I. Pérez-Conesa. «Comportamiento de ceras utilizadas en microfusión tras su calentamiento por microondas: técnica cascarilla cerámica». Técnica Industrial, 35-36 (2020). 


\begin{tabular}{lll}
\hline \multicolumn{2}{c}{ TABLA 1. RESUMEN POR COMPARATIVA DE LAS METODOLOGÍAS } \\
PARA EL PROCESADO TÉRMICO. ESQUEMA DE PLAN DE TRABAJO
\end{tabular}

des dieléctricas de las ceras sintéticas son similares y en cualquiera de los casos bajas y muy inferiores de las naturales. La elección de la cera roja de modelar para este estudio se justifica por encontrarse dentro de las ceras sintéticas, compuesto comúnmente utilizado en la técnica de joyería tradicional, además de emplearse también en otras técnicas de fundición artística. En pruebas preliminares se ha resuelto la hipótesis de que las bajas propiedades dieléctricas de las ceras sintéticas son mitigadas por las altas que caracterizan al molde para el procedimiento de descerado por MW (microondas) dado su alto porcentaje de agua.

Para preparar los ensayos, a cada una de las ceras se le ha practicado un molde cilíndrico de $30,3 \times 60 \mathrm{~mm}$ con pasta cerámica para fundir (Revestimiento WHIP MIX PRIMA-CAST) 100:40 partes de agua, siguiendo el procedimiento de elaboración convencional. El material utilizado para el encofrado del molde, en condiciones habituales, sería de metal para soportar sin problemas las altas temperaturas, pero en el caso del microondas, a priori se producirían daños en el dispositivo $^{30}$. Por lo que se ha sustituido por PVC, en este caso de $40 \times 70 \mathrm{~mm}$ de formato

30 J. Menéndez y A. H. Moreno. Aplicaciones... 
y con 6 perforaciones a lo largo de la superficie para facilitar la salida de vapores. Para desencofrarlo, y así poder introducir el molde en la mufla tras su descere, se ha dividido en dos valvas, que se fijan entre sí con una goma elástica, para mantener presionado el combinado, y soportar la fuerza ejercida por el vapor de agua o la expansión de la cera durante el proceso de descere.

La recogida de cera (figura 2) se realiza en una bandeja de forma cuadrada construida con cascarilla cerámica de $100 \mathrm{~mm}$ de lado y $10 \mathrm{~mm}$ de altura, ya que este material ha demostrado ser transparente a las $\mathrm{MW}^{31}$, por lo que no interfiere en la capacidad de absorción de ondas de los elementos sometidos a estudio.

Un segundo grupo de pruebas lo constan 10 muestras realizadas del mismo modo descrito, con la diferencia de que el modelo de cera se complica en su configuración al incorporársele 4 ramificaciones, generando así una forma cactus cuyo nombre asignado es Forma B, con un peso de promedio de 2,92 g. Estas pruebas servirán para la verificación de la primera parte del proceso (descere y secado) y de estudio del diseño de mufla para MW incluyendo el desarrollo de una curva de quemado que será testada mediante la colada del metal, realizada inmediatamente al procesado térmico con latón fundido a $940^{\circ} \mathrm{C}$. En el proceso no se emplearán fuerzas adicionales a la ejercida por la presión metastática por propia gravedad: por lo que en este caso la ignición y el elemento de metal obtenido determinan la calidad del quemado.

Para procesar las pruebas, se han empleado metodologías que permitan despejar variables con el fin de estudiar el comportamiento en los moldes dado únicamente por la variable tiempo/potencia empleada. La colocación de las muestras se realiza en la zona central de la cavidad del microondas, concretamente, el modelo de horno utilizado ha sido el microondas OK OMW 2214 B con 700W, de rendimiento máximo. Este dispositivo ofrece variantes de emisión de microondas, pudiendo oscilar su potencia para diseñar las curvas de aplicación: siendo LOW el $15 \%$, M.LOW un 30\%, M. 50\%, M. HIGH 75\% y HIGH el $100 \%$ de rendimiento de la potencia superior.

El tiempo transcurrido desde el fraguado al procesado ha sido de una hora. Se regula el tiempo y el rendimiento buscando una curva de temperatura suave y paulatina para que el molde vaya liberando la humedad a medida que se eleva su temperatura, equilibrando las propiedades dieléctricas dada la deshidratación y aumento de potencia para reproducir un descerado no violento tal como dicta la técnica convencional. Una vez que se han perdido las propiedades dieléctricas por el desarrollo de la deshidratación del molde, se estudia la curva aplicada para conseguir el aumento de temperatura requerida entre $700^{\circ} \mathrm{C}-800^{\circ} \mathrm{C}$, utilizando el mismo horno microondas de características en cuanto a potencia meramente convencionales.

Para el cumplimiento de este requerimiento se ha fabricado una doble mufla para MW, con un formato exterior de $150 \times 150 \times 150 \mathrm{~mm}$ y un espesor interno de $20 \mathrm{~mm}$, utilizando un ladrillo refractario de baja densidad compuesto de Al2O3

${ }^{31}$ F. Moreno Candel, F.F. Hernández e I. Pérez-Conesa. Comportamiento... 
67\% y SiO2 30,05\% (Ladrillos Ligeros Clas. Astm). Éste se ha dividido en dos y se le ha practicado una cavidad central, de modo que, al enfrentar los dos fragmentos, generen una cámara cilíndrica de $70 \mathrm{~mm}$ de diámetro y $70 \mathrm{~mm}$ de altura, que alberga un tubo también cilíndrico fabricado con material electromagnético de $0,5 \mathrm{~mm}$ de espesor, $68 \mathrm{~mm}$ de diámetro y $68 \mathrm{~mm}$ de altura, que contendrá las muestras. Este elemento susceptor de microondas está compuesto por una mezcla de carburo de silicio verde $(400 \mathrm{~mm})$ aglutinado con un $5 \%$ de bentonita y el mínimo porcentaje de agua necesario para generar una pasta moldeable para su fabricación manual. El principio de funcionamiento se basa en que el elemento más absorbente a las microondas del combinado $(\mathrm{SiC})$ transferirá por conducción térmica la temperatura al molde ayudado por la retención calorífica de la alúmina y el sílice, que además se mostrarán transparentes a las ondas, puesto que sus propiedades dieléctricas son muy bajas y, por tanto, no se calienta presentándose inerte al proceso ${ }^{32}$. Esta idea del uso de la mufla de calentamiento parte, en cuanto al concepto de susceptor, obviamente de la aportación de David Reid, siguiendo su idea original de encerrar el molde en una mufla, y no integrando el material susceptor, como Albaladejo en la composición del molde de cáscara, en nuestro caso en la composición del revestimiento de yeso. David Reid la utilizó para fundir el metal, nosotros para calentar el molde de yeso en su segunda fase, para la deshidratación.

Durante todo el estudio, los resultados vienen analizados por la medición de pesos, temperaturas y observación de fisuras o desmoronamientos en el molde. Los datos térmicos se han recogido a través de sistemas de infrarrojos, por medio de un termómetro láser (F-62 Max-plus) y una cámara para capturas de termografías (FlukeTis20) colocadas a $35 \mathrm{~cm}$ de la muestra. Por otro lado, la humedad se registra con el higrómetro Stanley 0-77-030.

\section{RESULTADOS}

La pasta refractaria actúa como un yeso, en primera instancia fragua apelmazando contra el encofrado, durante la primera fase de aumento de temperatura, hasta los $116^{\circ} \mathrm{C}$, donde se pierde la mayor parte de cera y agua, el PVC ha resultado idóneo, puesto que no absorbe microondas, se calienta principalmente por conducción térmica desde el molde, mostrándose transparente a las microondas ${ }^{33}$. Debido a que el PVC a los $80^{\circ} \mathrm{C}$ comienza a fundirse (Electrome, s.f.), cuando la cera se ha evacuado en su mayor parte, se retira el PVC, para volver a introducir el molde en la mufla.

32 J.Á. Menéndez Díaz y Á.S. Hernández Moreno. «Secado industrial con energía microondas». En Aplicaciones industriales del calentamiento con energía microondas, J.M. Menéndez (315). Cotopaxi: Universidad Técnica de Cotopaxi, 2017.

${ }^{33}$ R. Mujal Rosas, J. Orrit Prat, X. Ramis Juan, M. Marín Genesca y A. Rahhali. «Características dieléctricas de diversos polímeros (PVC, EVA, HDPE, y PP) reforzados con neumáticos fuera de uso (GTR)». Afinidad LXVIII (554), (2011): 263-273. 


\begin{tabular}{|c|c|c|c|c|c|c|c|c|}
\hline Muestra & $\begin{array}{c}\text { LOW } \\
15 \%\end{array}$ & $\begin{array}{c}\text { M. LOW } \\
30 \%\end{array}$ & $\begin{array}{c}\text { M. } \\
50 \%\end{array}$ & $\begin{array}{c}\text { M. HIGH } \\
75 \%\end{array}$ & $\begin{array}{c}\text { HIGH } \\
100 \%\end{array}$ & $\begin{array}{c}\text { Tiempo } \\
\text { TOtal }\end{array}$ & $\begin{array}{l}\text { Temp } \\
\text { Max. }\end{array}$ & Resultado \\
\hline $1 \mathrm{~A}$ & 5 ' & $3^{\prime}$ & $3^{\prime}$ & $0^{\prime}$ & $0^{\prime}$ & $11^{\prime}$ & $\mathrm{X}$ & Estalla 3’M. \\
\hline $2 \mathrm{~A}$ & 5 & 5 & $3^{\prime}$ & $0^{\prime}$ & $0^{\prime}$ & $13^{\prime}$ & $\mathrm{X}$ & Estalla 3’M. \\
\hline $3 \mathrm{~A}$ & $7^{\prime}$ & 5 & $2^{\prime}$ & $0^{\prime}$ & $0^{\prime}$ & $14^{\prime}$ & $\mathrm{X}$ & Estalla 2’M. \\
\hline $4 \mathrm{~A}$ & 10 & 5 & $1^{\prime}$ & $0^{\prime}$ & 0 ' & 16 & $88^{\circ} \mathrm{C}$ & Desprende cera con yeso. \\
\hline $5 \mathrm{~A}$ & $10^{\prime}$ & $7^{\prime}$ & $0^{\prime}$ & 0 ' & 0 ' & $17^{\prime}$ & $87^{\circ} \mathrm{C}$ & Desprende cera con yeso. \\
\hline $6 \mathrm{~A}$ & 10 & $5^{\prime}$ & $5^{\prime}$ & $0^{\prime}$ & $0^{\prime}$ & 20 & $85^{\circ} \mathrm{C}$ & Desprende cera con yeso. \\
\hline $7 \mathrm{~A}$ & $10^{\prime}$ & $10^{\prime}$ & $2^{\prime}$ & $0^{\prime}$ & 0 ' & $22^{\prime}$ & $88^{\circ} \mathrm{C}$ & Desprende cera con yeso. \\
\hline $8 \mathrm{~A}$ & 15 & 12 & 1' & 0 ' & 0 ' & $28^{\prime}$ & $87^{\circ} \mathrm{C}$ & Desprende yeso. Descere parcial. \\
\hline $9 \mathrm{~A}$ & 20 ' & $12^{\prime}$ & $1^{\prime}$ & 0 ' & $0^{\prime}$ & $33^{\prime}$ & $106^{\circ} \mathrm{C}$ & Descere total sin deterioros. \\
\hline $10 \mathrm{~A}$ & $20^{\prime}$ & $10^{\prime}$ & $1^{\prime}$ & 0 ' & 0 ' & $31^{\prime}$ & $95^{\circ} \mathrm{C}$ & Descere total sin deterioros. \\
\hline $11 \mathrm{~A}$ & $20{ }^{\prime}$ & $7^{\prime}$ & $1^{\prime}$ & 0 ' & 0 ' & $28^{\prime}$ & $109^{\circ} \mathrm{C}$ & Descere total sin deterioros. \\
\hline $12 \mathrm{~A}$ & $20^{\prime}$ & 5 & 5 & 0 ' & $0^{\prime}$ & 30 & $92^{\circ} \mathrm{C}$ & Descere total sin deterioros. \\
\hline \multicolumn{9}{|c|}{ Pruebas de Repetición } \\
\hline $13 \mathrm{R}$ & 20 & 5 & 5 & $0^{\prime}$ & $0^{\prime}$ & $30^{\prime}$ & $116^{\circ} \mathrm{C}$ & Descere total sin deterioros. \\
\hline $14 \mathrm{R}$ & 20 ' & 5 & 5 & $0^{\prime}$ & 0 ' & $30^{\prime}$ & $98^{\circ} \mathrm{C}$ & Descere total sin deterioros. \\
\hline $15 \mathrm{R}$ & $20^{\prime}$ & 5 & 5 & 0 ' & 0 ' & $30^{\prime}$ & $106^{\circ} \mathrm{C}$ & Descere total sin deterioros. \\
\hline
\end{tabular}

La sensibilidad requerida en la primera meseta ha sido en el modo LOW (15\% de potencia), quedando establecidos un mínimo de 20 minutos, ya que, como se observa en la tabla 1 , si en una primera instancia se procede a aumentar la potencia, el aumento súbito de temperatura provoca fisuras y, en consecuencia, se desmorona o estalla algún fragmento debido a la ebullición del agua contenida en la pasta o a la expansión brusca de la cera. Por lo que, solo una vez transcurridos 20 minutos en LOW, es posible aumentar a las siguientes potencias, estableciéndose como límite para el PVC no más de $116^{\circ} \mathrm{C}$ en el molde, dado que ésta ha sido la temperatura promedia registrada en los casos donde el PVC empieza a derretirse. Es en este momento cuando finaliza la primera etapa de secado y descerado y, como hemos comentado anteriormente, se interviene para retirar el encofrado, extraer la cera fundida del interior del horno microondas y proceder con la mufla susceptora. La humedad absoluta del molde se mide antes del procesado, siendo de un $2.0 \%$ el promedio obtenido y tras su descere, presenta un promedio de humedad de $0.6 \%$ (rango de medición: ca. 0,2\% hasta 2.0\%).

Con estos resultados, se determina como diseño efectivo de curva tiempo/ potencia: 20 ' LOW 15\%, 5' M.LOW 30\%, 5' M 50\% para un cilindro de $3,30 \times 60 \mathrm{~mm}$. En estos resultados, se aprecia que, a partir de la muestra $12 \mathrm{~A}$, manteniendo esta curva no aparece polvo de yeso en la cera recolectada ni se producen 


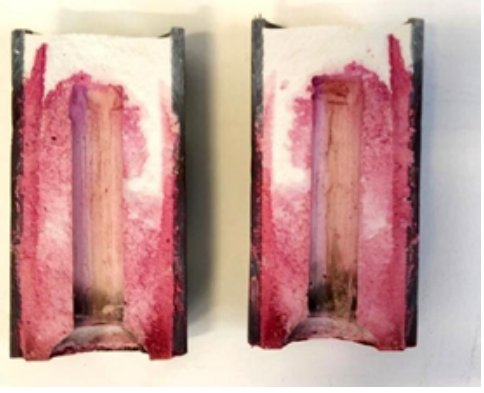

Fig. 2. Sección de la Muestra 6A.

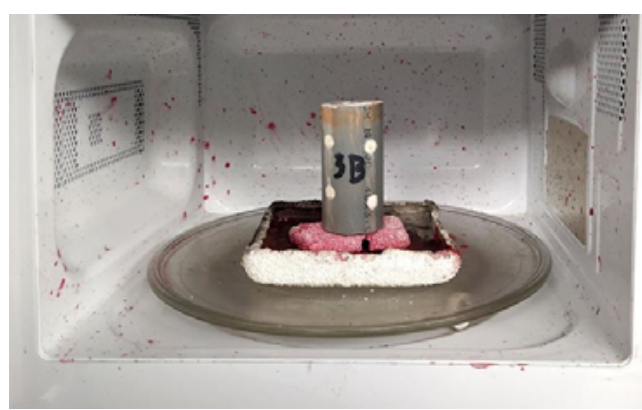

Fig. 3. Disposición en la cavidad donde puede apreciarse la bandeja recolectora de cera de cáscara cerámica. Muestra 3B.

\begin{tabular}{|c|c|c|c|c|}
\hline \multicolumn{5}{|c|}{ TABLA 3. RESULTADOS PESOS DE CERA MUESTRAS (B-1/B-10) } \\
\hline Muestra & $\begin{array}{l}\text { Peso de } \\
\text { CERA (G.) }\end{array}$ & $\begin{array}{l}\text { Peso sin } \\
\text { Cera (G.) }\end{array}$ & $\begin{array}{c}\text { Peso Cera } \\
\text { ReCOLECTAdA (G.) }\end{array}$ & $\%$ Descere \\
\hline B.1 & 3 & 74,9 & 2,3 & $76.66 \%$ \\
\hline B. 2 & 2,9 & 77,3 & 2,2 & $75,86 \%$ \\
\hline B. 3 & 2,9 & 75,6 & 2,6 & $89.65 \%$ \\
\hline B. 4 & 2,8 & 77,9 & 2,6 & $92.85 \%$ \\
\hline B. 5 & 3 & 77,3 & 3 & $100 \%$ \\
\hline B.6 & 3,2 & 76,8 & 3 & $93.75 \%$ \\
\hline B.7 & 2,8 & 75,9 & 2,2 & $78.57 \%$ \\
\hline B. 8 & 2,7 & 76,1 & 2,6 & $96.29 \%$ \\
\hline B. 9 & 2,8 & 76,2 & 2,6 & $92,85 \%$ \\
\hline B. 10 & 3,1 & 78,3 & 2.8 & $90,32 \%$ \\
\hline
\end{tabular}

estallidos, consiguiendo descerar el molde sin deteriorarlo. Las figuras 1 y 2 ilustran el formato y disposición de las muestras.

Tras calcular el peso antes y después de su proceso térmico, en la tabla 3 se deduce un descerado promedio de $88,68 \%$.

La siguiente etapa es alcanzar los $750^{\circ} \mathrm{C}$ para eliminar el resto de las partículas de cera y cocer el yeso, pero a medida que aumenta la temperatura y se desarrolla la deshidratación del molde, consecuentemente también decrecen las propiedades dieléctricas, por lo que se debe aumentar el potencial de calentamiento. Llevar al máximo rendimiento la potencia del $\mathrm{MW}$ no es suficiente, el material refractario que compone el molde se muestra transparente a las microondas y supone un riesgo para la unidad, propiciando un sobrecalentamiento que provoca daños en su magnetrón. En un primer momento, se estudió añadir susceptores a la composición del 


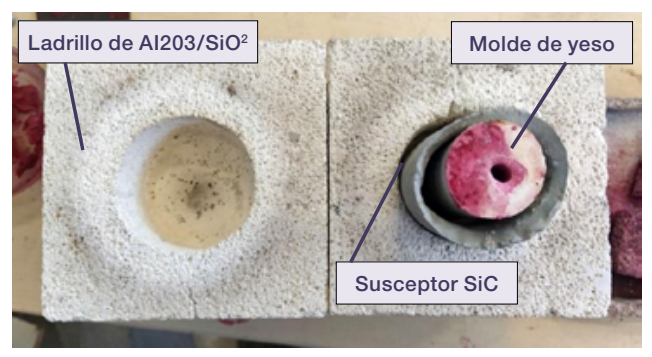

Fig. 4. Partes que componen la Mufla MW.

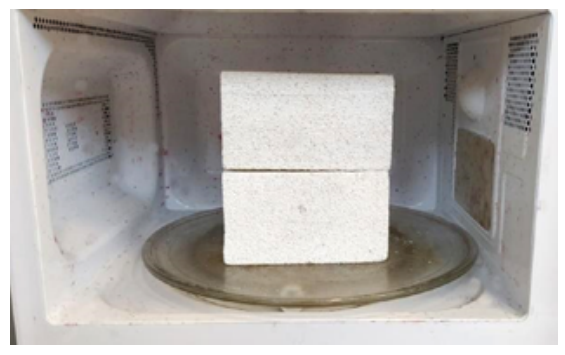

Fig. 5. Mufla cerrada en el interior del microondas.

yeso, pero se descartó esta medida por los resultados obtenidos cuando se añadieron en la mezcla de la cáscara cerámica o como estuco exterior del molde, porque generaba un calentamiento demasiado rápido y descontrolado, produciéndose, en los primeros segundos, exclusivamente en la zona de aplicación. La mejor alternativa la constituye la conductividad térmica, provocada por un aislamiento térmico y una sección de susceptor considerable. Para propiciar estas condiciones se diseñaron varias muflas, siendo descartadas por su funcionamiento poco efectivo. Finalmente, el diseño de la doble mufla para microondas (figura 3), descrita anteriormente y que se ensaya en el grupo de muestras con Forma B, obtuvo los resultados requeridos.

Como se observa en las figuras 4 y 5 , gracias al elemento susceptor de carburo de silicio la energía se concentrará en la masa perimetral que circunda el molde hasta su núcleo por estar aislada gracias al ladrillo refractario.

De este modo, las 10 muestras de "Forma B», desceradas y secadas, se van testando, hasta dar con una curva de temperatura adecuada que no genere anomalías en el molde. La búsqueda radica en imitar una curva de calentamiento que no resulte agresiva para el negativo y que alcance $\operatorname{los} 750^{\circ} \mathrm{C}$. Como se observa en la gráfica 1 , las primeras muestras obtuvieron resultados fallidos, donde el molde no alcanzaba la temperatura necesaria. La muestra B.5 presenta una curva adecuada para el proceso, establecida en L.15\%:2'/ M. L30\%:2'/ M.50\%:3'/ M.H.75\%:4'/ H100\%: 6'.

Este resultado se repite en las restantes (B.6-V a la B.10), obteniendo resultados adecuados sin fisuras o desprendimientos.

Por último, con las muestras procesadas B.5 a B10 (figuras 6 y 7), se efectúa una colada de latón, fundido en horno de gas durante el procesado del molde, efectuando una colada por gravedad en el molde, que al no extraerlo del combinado refractario se mantiene a una temperatura aceptable alrededor de los $600^{\circ} \mathrm{C}$. De esta manera se verifica la capacidad de registro del negativo, si se propician combustiones por un quemado insuficiente, así como la capacidad del molde para soportar la presión metalostática del llenado. En este proceso el molde debe estar lo suficientemente caliente para facilitar el recorrido del metal a través de los conductos más pequeños. La temperatura ideal de colada para el latón son $600^{\circ} \mathrm{C}$, suponiendo ries- 


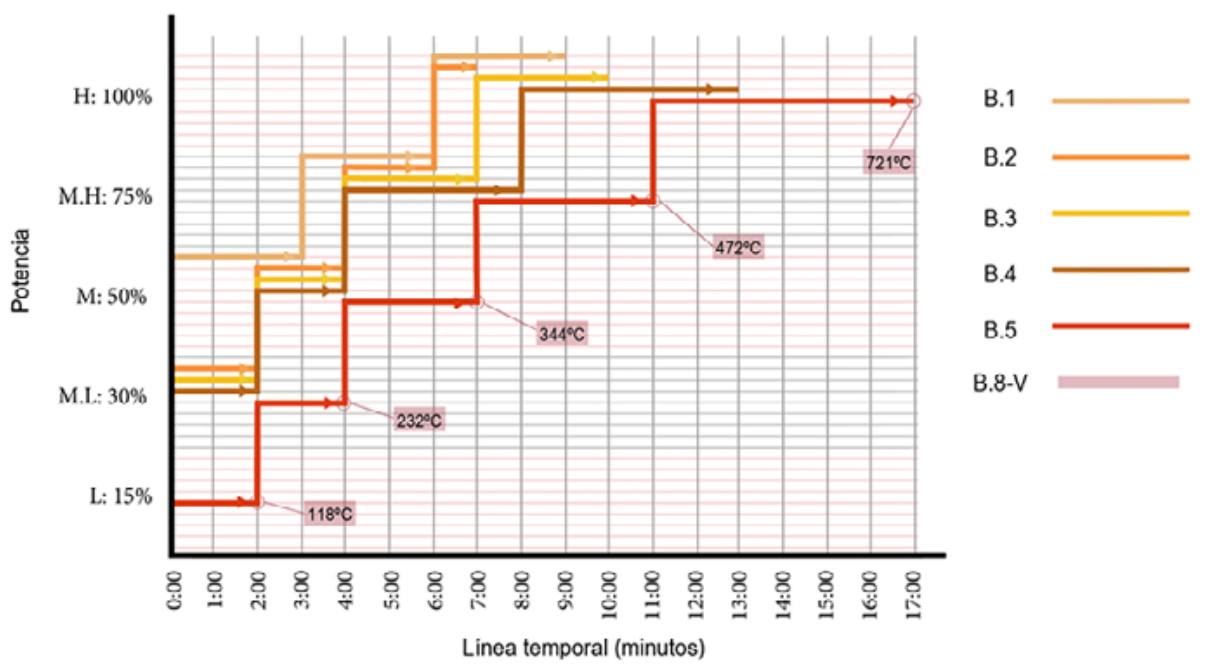

Gráfica 1. Curva de Temperatura/Tiempo/Potencia Muestras (B).

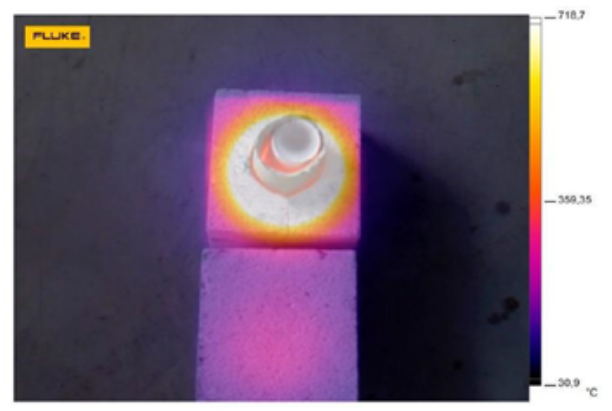

Fig. 6. Termografía de la Muestra B.9.

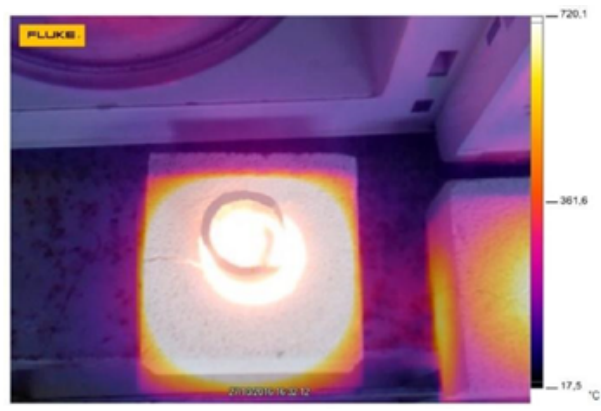

Fig. 7. Termografía de la Muestra B.10.

gos en temperaturas inferiores o superiores ${ }^{34}$. En este caso, el ladrillo de alúmina permite la manipulación sin pinzas metálicas, al estar a una temperatura muy inferior a la del molde; además, su refractariedad lo mantiene caliente para la colada (figura 10), descendiendo a los $600^{\circ} \mathrm{C}$, aproximadamente durante el tiempo de mani-

34 K.A. Krekeler. Microfusión... 

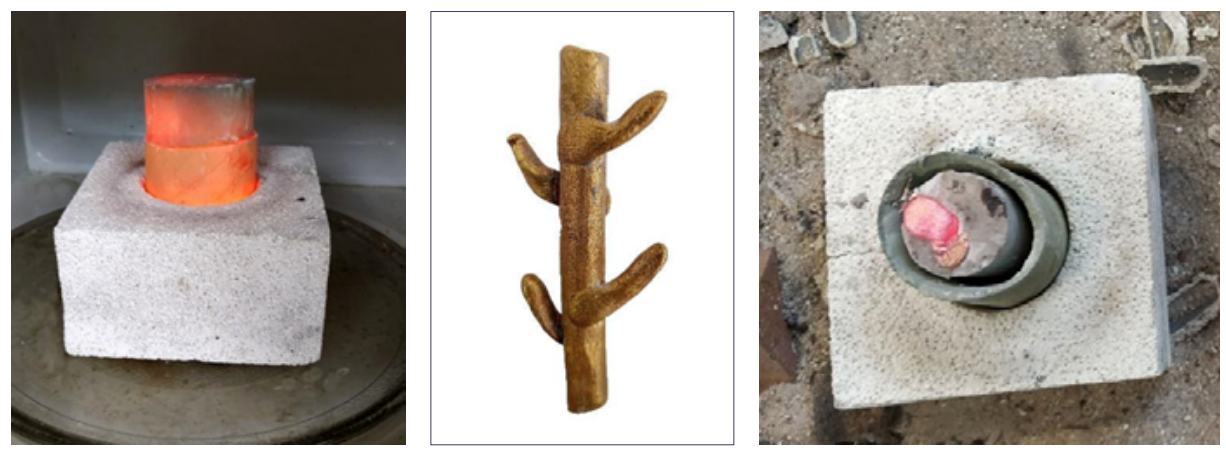

Fig. 8, 9 y 10 de izquierda a derecha. Mufla incandescente dentro del MW. Pieza

«Forma B», reproducida en latón. Colada de metal por gravedad en el molde caliente dentro de la mufla abierta.

pulado. Aunque se han producido leves reacciones por la aparición de llama, ésta ha permitido la continuidad de la colada. Se han obtenido 5 muestras en latón fieles a los modelos en cera primitivos. Los moldes tras el quemado han mostrado partes grisáceas que denotan restos de humedad, detalles que ameritan estudio dado que denotan un quemado incompleto. Sin embargo, no ha habido desmoronamientos ni escapes en el vertido, lo cual denota que no ha habido fisuras internas producidas durante el proceso térmico, lo cual significa que el calentamiento ha sido suficientemente escalonado para el deshidratado. Tampoco hay modificaciones aparentes en el registro de superficie (figura 9).

\section{CONCLUSIONES}

Tras las pruebas realizadas, se determina que la cantidad de agua utilizada en la realización de los moldes cilíndricos de joyería tradicional supone un aliado perfecto en el calentamiento por MW (microondas), requiriendo un escalamiento de potencia aplicado convenientemente al formato de la muestra. La hipótesis principal de esta investigación emerge de la necesidad de imitar una curva de calentamiento adaptada a estas condiciones y concluido el estudio se ha resuelto una curva eficaz diseñada en tiempo/potencia apta para cilindros de $3,30 \times 60 \mathrm{~mm}$ formados en pasta refractaria para la reproducción de muestras de 3 a 5 g de cera con un $90 \%$ de éxito aproximadamente en 30 minutos de procesado. El encofrado de PVC ha resultado un material idóneo para el proceso $\mathrm{MW}$, dado que la cera licúa a los $80^{\circ} \mathrm{C}$ y hasta que el molde no alcanza una temperatura cercana a los $100^{\circ} \mathrm{C}$, éste no se ve dañado, obteniendo un molde descerado, semiseco y sin fisuras antes de tener que retirar el plástico para que no se queme.

Si bien es cierto que el calentamiento inicial es apto únicamente, para llevar a cabo la primera parte del proceso, en el que la cera y el molde deben evacuarse 
y secarse, respectivamente, surge una dificultad: a medida que el molde va deshidratándose, las propiedades dieléctricas disminuyen proporcionalmente, no siendo suficientes para alcanzar la temperatura de quemado. Para superar este hándicap, se ha estudiado una solución mediante el recurso del «calentamiento híbrido», diseñando una mufla de carburo de silicio, alúmina y sílice que posibilita la capacidad térmica hasta conseguir comunicar al molde $721^{\circ} \mathrm{C}$ por conducción térmica con un microondas convencional de $700 \mathrm{w}$, basada en una solución utilizada por David Reid para fundir microfusión de cascarilla en un horno MW. De igual modo, ha sido necesario diseñar una curva de aplicación progresiva que no produzca fisuras en el molde, estableciendo 17 minutos de potencia escalonada para la obtención de fundiciones válidas, sin escapes de metal y teniendo una capacidad de registro fiel al original de cera.

Con los resultados del estudio se valida la posibilidad de producir pequeñas piezas de metal procesado térmicamente el revestimiento de joyería tradicional al poner en práctica un procedimiento de adaptación a la técnica que además servirá de sustrato para proseguir el estudio de adaptación que permita valorar las ventajas que pueden ofrecer los sistemas mediante $\mathrm{MW}$, como son la rebaja de tiempos y la simplificación de las infraestructuras, y por lo tanto posibilitar la reducción de consumos y emisiones, facilitando con ello la producción de piezas en metal fundido en talleres docentes o profesionales.

Dada la novedad del procedimiento utilizado no se han podido abordar aspectos que resultan básicos para las pretensiones del trabajo, como son el aumento de tamaño de las muestras a procesar, la complejidad de los modelos de cera y la mejora de la calidad del quemado y que se proyectan para los estudios consiguientes.

La consecuencia más trascendente de este estudio ha sido el excelente comportamiento de las pastas durante el descere y secados iniciales, unido a la verificación del funcionamiento del prototipo de mufla, creando expectativas potenciales.

Dentro de estos objetivos, además de otros aspectos, se pretende subsanar carencias importantes que para estos formatos no han imposibilitado la fundición pero que se consideran esenciales como son la aplicación de temperaturas de mantenimiento para el consecuente desarrollo del quemado y la solución de encofrado apto para el mismo con MW. 


\section{REFERENCIAS}

Aguilar Galea, J.A. La enseñanza de la fundición artística en las facultades de Bellas Artes Españolas». Tesis doctoral. Universidad de Sevilla, 2000.

Albaladejo González, J.C. «Fundición a la cera perdida: Cellini y la magnetita». Bellas Artes, 4, (2006): 13-28.

Albaladejo González, J.C., Del Pino, S. y Pérez Conesa, I. «Fundición artística: descere por microondas». Fundidores, 226 (2016): 19-21.

Álvarez, A.C. Sinterización en aplicaciones industriales del calentamiento con energía microondas. Ecuador: Universidad Técnica de Cotopaxi, 2017.

Benavente, J. La fundición a la cera perdida: Microfusión. Barcelona: Alsina, 1992.

Buenavente Martínez, R. Desarrollo de materiales cerámicos avanzados con altas prestaciones mediante técnicas no convencionales. Valencia: Universidad Politécnica de Valencia, 2015.

Cárdenas, C., Restrepo, R., García Sucerquia, J., Marín, J. y García, C. Las microondas como una alternativa para el secado de materiales cerámicos tradicionales. Universidad Simón Bolívar, 2009.

Carolina, C. «Las microondas como una alternativa para el secado de materiales cerámicos tradicionales». Revista Latinoamericana de Metalurgia y Materiales (2009): 428-431.

Chhillar, P., Agrawal, D. y Adair, J.H. «Sintering of Molybdenum metal powder using microwave energy». Powder Metallurgy, 51(2), (2008): 182-187.

Corredor, J. Técnicas de fundición artística. Universidad de Granada, 1997.

Electrome, S. (s.f.). www.electrocome.com. https://www.electrocome.com/p-1-36/POLICLORURO-DE-VINILO---PVC.htm.

Groover, M. «Fundamentos de manufactura moderna». México, 243 (1997).

Juan Aguilar, J.R. Producción de Carburo de Silicio utilizando Microondas como fuente de energía, 2014.

Krekeler, K.A. Microfusión. Fundición con modelo perdido. Gustavo Gili, 1971.

Martínez, R.B. "Desarrollo de materiales cerámicos avanzados con altas prestaciones mediante técnicas no convencionales de sinterización: microondas». Tesis Doctoral. Universidad Politécnica de Valencia, 2015.

Menéndez Díaz, J.Á. y Hernández Moreno, Á.S. «Secado industrial con energía microondas», en Aplicaciones industriales del calentamiento con energía microondas, J.M. Menéndez, (315). Cotopaxi: Universidad Técnica de Cotopaxi, 2017.

Menéndez, J. y Moreno, A.H. «Aplicaciones del calentamiento con energía microondas», en Secado industrial con microondas (85). Cotopaxi: Ed. Universidad Técnica de Cotopaxi, 2017.

Moreno Candel, F., Hernández, F.F. y Pérez-Conesa, I. «Comportamiento de ceras utilizadas en microfusión tras su calentamiento por microondas: técnica cascarilla cerámica». Técnica Industrial, 35-36. (2020).

Mujal Rosas, R., Orrit Prat, J., Ramis Juan, X., Marín Genesca, M. y Rahhali, A. «Características dieléctricas de diversos polímeros (PVC, EVA, HDPE, y PP) reforzados con neumáticos fuera de uso (GTR)». Afinidad LXVIII (554), (2011): 263-273. 
Pérez Conesa, I. «Técnica del descere por microondas: identificación, cuantificación y valoración de los susceptores». Tesis doctoral, Universidad de La Laguna, 2017.

Plaza González, P.J. Control de la temperatura en sistemas de calentamiento por microondas. Valencia: UPV, 2015.

Prado Gonjal, J. y Morán, E. Sintesis asistida por microondas de sólidos inorgánicos, 2 (2011): 129-136.

Ramírez Gil, F.J. «Comparación entre las técnicas convencionales y las microondas para la conformación y secado de materiales cerámicos fabricados por colado». Colombia 16 (2010).

ReID, D. «El primer vaciado fundido (en plata) por microondas se realizó en 1993. Se demostró la técnica en una conferencia sobre microondas en el St. John's College de Cambridge en 1995». (2018). https://hackaday.com/2005/01/24/microwave-oven-foundry/n.

Segura de Jesús, Y. y Carbaja, G. «El uso de radiación de microondas para la síntesis de Nanopartículas». Revista de Innovación Sistemática, 1(2), (2017): 46-56.

Sorroche Cruz, A., Lozano Rodríguez, I., Durán Suárez, J., Peralbo Cano, J. y Bellido Márquez, C. «Mejora de los métodos de trabajo en la fundición escultórica con modelos de poliestireno expandido». Revista Técnica Industrial, 1-2 (2009).

Valdez Nava, Z. Sinterización de manganitas Ni Fe empleando microondas como fuente de energía. León: Universidad Autónoma de Nuevo León, 2005. 
